\title{
El ángulo escafolunar, otro factor pronóstico de la seudoartrosis del escafoides
}

\author{
A. García Olea ${ }^{1}$, A. Dudley Porras ${ }^{1}$, E. González Fernández ${ }^{2}$, \\ D. Martínez VéLeZ ${ }^{2}$ \\ Hospital Universitario la Princesa. Madrid \\ (1) Médico Adjunto, Servicio de Traumatología y Cirugía Ortopédica, Unidad de Mano \\ (2) Médico Residente, Servicio de Traumatología y Cirugía Ortopédica, Rotante Unidad de Mano.
}

Correspondencia:

Dr. Ángel García Olea

Hospital Universitario Puerta de Hierro

C/ Manuel de Falla, 1

28220 Majadahonda. Madrid. España

Teléf.: 911916538

e-mail: garcialerma@msn.com

Se realiza un estudio retrospectivo de las seudoartrosis del escafoides carpiano, que no afectan al polo proximal, intervenidas en el HU La Princesa entre enero de 1996 y junio de 2008.

Material y método: Se estudiaron 40 pacientes intervenidos según las técnicas de Matti-Russe o Fisk-Fernández. En ninguno de los casos se trataba de reintervenciones, no existían cambios degenerativos del carpo, ni datos radiológicos de necrosis del fragmento proximal. Diez pacientes fueron tratados según la técnica de Matti-Russe y los otros 30 mediante la de Fisk-Fernández. Se realizó un protocolo de trabajo que estudió: datos de filiación, tiempo transcurrido hasta la cirugía, ángulo escafolunar pre y postquirúrgico, estadificación según las clasificaciones de Alnot y patrón degenerativo SNAC, técnica quirúrgica, material de osteosíntesis, tiempo transcurrido hasta la consolidación, reincorporación a su trabajo y existencia de complicaciones.

Resultados y conclusiones: La consolidación ósea se consiguió en 36 casos (90\%) en un tiempo medio de 3 meses. Hay mayor número de pacientes tratados mediante la técnica de Fisk-Fernández; clasificados en los grupos de peor pronóstico radiológico (ángulo escafolunar alterado) y con mayor tiempo de evolución desde el traumatismo. No se apreciaron diferencias entre los materiales de osteosíntesis empleados. La técnica de Fisk-Fernández consigue
The authors make a retrospective study of carpal scaphoid non-unions not affecting the proximal pole and operated in Hospital La Princesa from january 1996 to june 2008.

Material and method: The authors evaluated 40 patients operated on, following the techniques described by Matti-Russe or Fisk-Fernández. None of our patients was a reoperation case or had carpal arthritic changes nor radiological data of proximal pole necrosis. Ten patients were operated as proposed by Matti-Russe and the other 30 as Fisk-Fernández. A study protocol was made in order to compile: filiation data, time elapsed from injury to surgery, scapholunate angle before and after surgery, Alnot classification and SNAC pattern grade, surgical technique, osteosynthesis material implanted, time elapsed until scaphoid union, return to work or not and complications occurred.

Results and conclusions: Scaphoid union occurred in 36 cases (90\%) in a mean time of 3 months. The greater group is that operated on following the technique proposed by Fisk-Fernández, those classified as worse radiologic prognosis (altered scapholunate angle) and longer lap of time from injury. No statistical difference was observed between different osteosynthesis materials. Fisk-Fernández technique improves scapholunate angle but not the time necessary to scaphoid union. 
mejorar el ángulo escafolunar, pero no un menor tiempo de consolidación.

Palabras clave: Seudoartrosis de escafoides, injerto corticosponjoso, técnica de Fisk-Fernández, técnica de Matti-Russe, ángulo escafolunar.

Key words: Scaphoid non-union, corticocancellous bone graft, Fisk-Fernández technique, Matti-Russe technique, scapholunate angle.

Rev. Iberam. Cir. Mano - Vol. 38・Núm. 1 Mayo 2010 (31-39)

\section{INTRODUCCIÓN}

T as fracturas del escafoides son las más frecuentes del carpo. Un 2 ó 3\% de las fracturas del escafoides no son diagnosticadas en el traumatismo inicial al realizar unas Rx estándar ${ }^{1}$. A pesar de una adecuada inmovilización y atención médica especializada inmediata, diversos autores reflejan una incidencia de seudoartrosis del escafoides carpiano que oscila entre el $5 \mathrm{y}$ el $15 \%{ }^{1-5}$.

Pocas seudoartrosis de escafoides (SE) permanecen estables y asintomáticas tras 10 años de evolución. Existen múltiples estudios que demuestran que la historia natural de las SE es la artrosis con restricción del balance articular ${ }^{1-5}$. Por ello, el tratamiento habitual de la mayoría de las SE consiste en reducción abierta, aplicación de injerto óseo y osteosíntesis. La presencia de cambios radiológicos, la localización del foco de seudoartrosis, la constatación de cambios adaptativos del carpo, la viabilidad vascular del fragmento proximal y otros factores como la edad y ocupación del paciente, determinarán la técnica a elegir. Los objetivos del tratamiento quirúrgico deben ser la consolidación ósea, el restablecimiento de la longitud y geometría normales del escafoides y corregir los posibles cambios adaptativos del carpo.

\section{MATERIAL Y MÉTODO}

Se realiza un estudio retrospectivo de las seudoartosis del escafoides (SE) carpiano que no afectan al polo proximal intervenidas en el HU La Princesa entre enero de 1996 y junio de 2008, con el objetivo de conocer los resultados del tratamiento quirúrgico mediante injerto corticoesponjoso de cresta ilíaca.

A partir de un listado de 63 pacientes diagnosticados de SE, se estudiaron de forma retrospectiva 40 pacientes que se ajustaban a los condicionantes que se exponen a continuación. En ninguno de los casos se trataba de reintervenciones, la localización del foco de SE fue siempre el tercio medio, no existían cambios degenerativos del carpo de suficiente entidad en las Rx convencionales ni datos radiológicos de necrosis del fragmento proximal. En todos los casos se ha realizado una resección del foco de seudoartrosis y colocación de injerto de cresta ilíaca según la técnica de Matti-Russe (M-R), o injerto trapezoidal intercalado según la técnica de Fisk-Fernández (F-F).

En el servicio, se aplica un algoritmo que Smith y Cooney publicaron en $1996^{6}$, en el que se recomienda el uso de tratamientos agresivos como artrodesis o carpectomías en el caso de SE avanzadas, la estiloidectomía cuando existan cambios degenerativos a dicho nivel, el injerto óseo vascularizado para las necrosis del polo proximal, el injerto óseo intercalado en los casos de inestabilidad y el injerto óseo anterior según técnica de M-R en los casos estables.

Se trataron un total de 40 pacientes, de los cuales 35 eran hombres (87.5\%). Si diferenciamos la muestra por técnicas; mediante la de M-R se intervinieron 10 pacientes, de los cuales 9 eran hombres. De los 30 restantes, 26 fueron hombres.

En todos los casos el injerto óseo fue obtenido de la cresta ilíaca y se refrescaron los fragmentos hasta obtener sangrado puntiforme del lado proximal. Siempre se utilizó un método de osteosíntesis, bien tornillo canulado (Herbert- 
Whipple en los primeros casos y posteriormente AO canulado de $3 \mathrm{~mm}$ ), bien agujas de Kirschner (aK). En 15 pacientes se realizó la osteosíntesis con aK (36\%). La utilización de aK en la mayoría de casos fue debido a dificultades técnicas o por necesidad de un curetaje amplio del fragmento proximal, lo que no permite una presa del tornillo que asegure una estabilidad suficiente.

También en todos los casos se realizó una inmovilización con férula-yeso durante 6-8 semanas. El seguimiento postquirúrgico y control $\mathrm{Rx}$ se realizó de forma protocolizada a los 10 días y a las 6-8, 12, 24, 36-38, 52 semanas y a los 3 años.

Se realizó un protocolo de trabajo que estudió las siguientes variables: datos de filiación del paciente; tiempo transcurrido desde el traumatismo hasta la cirugía; ángulo escafolunar pre y postquirúrgico en la Rx lateral de muñeca ${ }^{7}$; estadio según las clasificaciones de $\mathrm{Alnot}^{8}$ y patrón degenerativo $\mathrm{SNAC}^{10,11}$; técnica quirúrgica empleada; tipo de material de osteosíntesis; consolidación ósea o ausencia de consolidación; tiempo transcurrido hasta la consolidación; reincorporación a su trabajo habitual; y la existencia de complicaciones. Tabla I.

Debido a que una gran mayoría de casos no presentaban cambios artrósicos en las Rx, se utilizó la clasificación de Alnot.

Se realizó estudio estadístico de la muestra obtenida (ANOVA, t de Student. Significación $\mathrm{p}<0,05)$, analizándose los siguientes datos:

A. Distribución de los pacientes según gravedad-técnica quirúrgica, resultando un patrón de muestra acorde con el protocolo establecido.

B. Relación entre tiempo de evolución, gravedad de cambios radiológicos y tiempo de consolidación.

C. Relación entre ángulo escafolunar, nivel de gravedad de cambios radiológicos y tiempo de evolución.

D. Mejoría estadísticamente significativa o no del ángulo postquirúrgico.

\section{RESULTADOS}

De acuerdo con la literatura, y con el criterio del servicio, hay un mayor número de pacientes tratados mediante la técnica de F-F; que fueron los clasificados en los grupos de peor pronóstico. También cabe destacar el tiempo de evolución desde el traumatismo, mayor en los pacientes intervenidos según técnica de F-F, con una media de 40 meses frente a 15 en los pacientes del grupo M-R. Esta diferencia no es estadísticamente significativa; al igual que tampoco se pudieron confirmar diferencias estadísticamente significativas entre la gravedad de los cambios radiológicos y tiempos de evolución y consolidación de la SE.

Respecto de las mediciones del ángulo escafolunar en las Rx laterales de muñeca; se ha objetivado una mejoría estadísticamente significativa $(\mathrm{P}<0,05)$ al utilizar la técnica de F-F. Así, el ángulo medio preoperatorio fue de 62,47 $+/-13,20^{\circ}$ (intervalo $40-90^{\circ}$ ) y el postoperatorio fue de $53,33+/-9,94^{\circ}$ (intervalo $40-80^{\circ}$ ). Para la técnica de M-R el ángulo postoperatorio no mejoró. También existe diferencia estadísticamente significativa en la distribución de los pacientes según el ángulo escafolunar pre-quirúrgico y el nivel de gravedad de los cambios radiológicos, así como entre ángulo pre-quirúrgico y el tiempo de evolución.

La consolidación ósea se consiguió en 36 casos $(90 \%)$. Si diferenciamos según la técnica empleada, encontramos que la consolidación supone el $90 \%$ (1 fracaso) mediante la técnica de M-R y el 90\% (3 fracasos) para la de F-F. De los 36 casos considerados curados, en cuatro la consolidación se alargó más de cinco meses; y de estos últimos, tres casos fueron tratados mediante la técnica de M-R. Es por ello que se objetiva un tiempo medio de consolidación de 5 meses para la técnica de M-R frente a tres meses en la técnica de F-F. Esta diferencia no es estadísticamente significativa. Si considerásemos como fracasos los casos de consolidación retardada, a pesar de no necesitar nueva cirugía, los datos bajan a $80 \%$ de éxitos en el total, $60 \%$ para la técnica de M-R y $86,7 \%$ para la de F-F. En estos casos de retardo de consolidación, el lapso de tiempo considerado para aceptar la consolidación coincide con la realización de una TAC o Rx selectivas en las que se aprecien puentes óseos, aunque éstos no sean en todo el espesor del hueso, y las imágenes puedan resultar artefactadas por el material de osteosíntesis. 


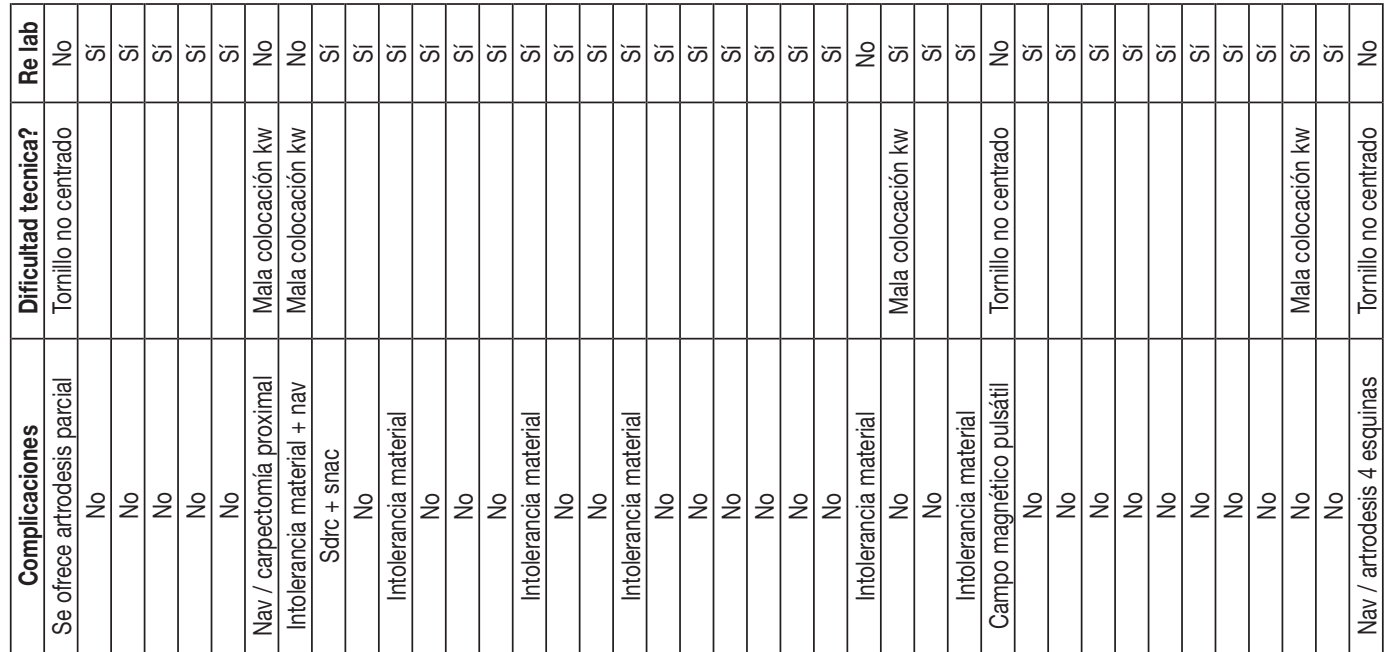

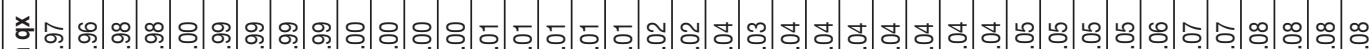

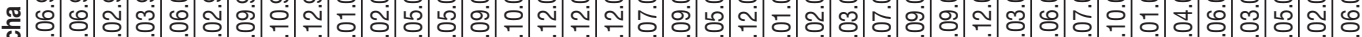

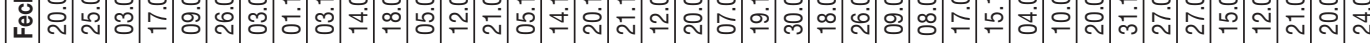
尊- 
TABLA II -RESUMEN TOtALES Y PARCIALES

\begin{tabular}{|l|c|c|c|}
\hline & Total & Matti-Russe & Fisk-Fernández \\
\hline $\mathrm{n}$ & 40 & 10 & 30 \\
\hline Edad media (años) & $26.95 \pm 8.30 \mathrm{R}=(15-44)$ & $26,20 \pm 7,95 \mathrm{R}=(17-40)$ & $27,20 \pm 8,53 \mathrm{R}=(15-44)$ \\
\hline Género & $35 \mathrm{H} / 5 \mathrm{M}$ & $9 \mathrm{H} / 1 \mathrm{M}$ & $26 \mathrm{H} / 4 \mathrm{M}$ \\
\hline Tiempo evolución previo (meses) & $33,59 \pm 38,75 \mathrm{R}=(3-192)$ & $15,35 \pm 14,75$ & $40,10 \pm 42,64$ \\
\hline Material osteosíntesis & Herbert (6) aK (15) AO (19) & Herbert (3) aK (3) AO (4) & Herbert (3) aK (12) AO (15) \\
\hline Clasificación alnot & $\mathrm{I}(16)$ IIA (12) IIB (12) & $\mathrm{I}(8)$ IIA (2) & I (8) IIA (10) IIB (12) \\
\hline Snac & $\mathrm{I}(12)$ & $55,5 \pm 6,43$ & $62,46 \pm 13,20$ \\
\hline Ángulo s-I prequirúrgico & $60,72 \pm 12,18$ & $55,5 \pm 5,50$ & $53,33 \pm 9,94$ \\
\hline Ángulo s-I postquirúrgico & $53,87 \pm 9,02$ & $\mathrm{SI}(9) \mathrm{NO}(1)$ & $3,06 \pm 0,99$ \\
\hline Consolidación & $\mathrm{SI}(36) \mathrm{NO}(4)$ & $4,94 \pm 3,62$ & Asintomáticos (26) \\
\hline Tiempo consolidación (meses) & $3,53 \pm 2,10$ & Asintomáticos (8) & Mejoría parcial (1) \\
\hline Evolución clínica & Asintomáticos (34) & Mejoría parcial (1) & Empeoramiento (3) \\
\hline & Mejoría parcial (2) & Empeoramiento (1) & No (22) \\
\hline & Empeoramiento (4) & No (8) & Intolerancia material (4) \\
\hline Complicaciones & No (30) & SDRC(1) \\
\hline & Intolerancia material(6) & Intolerancia material (2) & SDRC(0) \\
\hline
\end{tabular}

ABREVIATURAS: ÁNGULO S-L: Ángulo escafolunar; Herbert: Tornillo canulado Herbert; aK: Agujas de Kirschner; AO: Tornillo canulado AO 3mm; SDRC: Síndrome doloroso regional complejo

Por último, 34 pacientes quedaron asintomáticos y se reincorporaron a su puesto laboral (85\%), 2 tuvieron mejoría parcial y los 4 fracasos sufrieron empeoramiento clínico y radiológico. Tabla II.

\section{DISCUSIÓN}

Las fracturas del escafoides son las más frecuentes del carpo. Jupiter et al. ${ }^{1}$ refieren que las fracturas del escafoides suponen $2 / 3$ de los traumatismos del carpo y que un 2 ó $3 \%$ de las fracturas del escafoides no son diagnosticadas en el traumatismo inicial al realizar unas Rx estándar.

La incidencia de SE oscila entre el 5 y el $15 \%$ de las fracturas en dicho hueso ${ }^{1-5}$. Para algunos autores como Herbert, suponen hasta un $50 \%{ }^{12}$. Las fracturas consideradas inestables y/o desplazadas tienen un porcentaje mayor de evolución a seudoartrosis o consolidación con deformidad en joroba ${ }^{1,13,14}$. Pocas SE permanecen estables y asintomáticas tras 10 años de evolución. Lins- trom y Nyström, Mack et al., y otros ${ }^{2-5}$ intentaron correlacionar el tiempo de evolución de la seudoartrosis y los cambios radiológicos. Frente a cirujanos como Herbert ${ }^{12}$, que plantea como poco ético realizar un estudio con una cohorte de no tratados, encontramos autores como Ruby et al. ${ }^{4}$ quienes publican una serie de 56 seudoartrosis no tratadas en 55 veteranos en los que las patologías con más de 5 años desarrollaron una artrosis de muñeca. Los primeros autores que definieron una clasificación del patrón artrósico en la muñeca fueron Watson y Ballet ${ }^{9}$. Sin embargo, fueron las publicaciones de Krakauer et al. ${ }^{10} \mathrm{y}$ de Morimoto et al. ${ }^{11}$ las que establecieron el patrón degenerativo SNAC y más concretamente de las SE del tercio medio, que son más rápidamente sintomáticas y evolucionan más rápidamente.

Varias publicaciones reconocen que el porcentaje de éxito del tratamiento quirúrgico de las SE depende de factores como profesión de alta demanda funcional, presencia de cambios degenerativos y adaptativos del carpo, localización del foco de seudoartrosis y patología de más de cinco años de evolución ${ }^{1,13,14,15}$. También se relaciona una SE inveterada, y por tanto con 

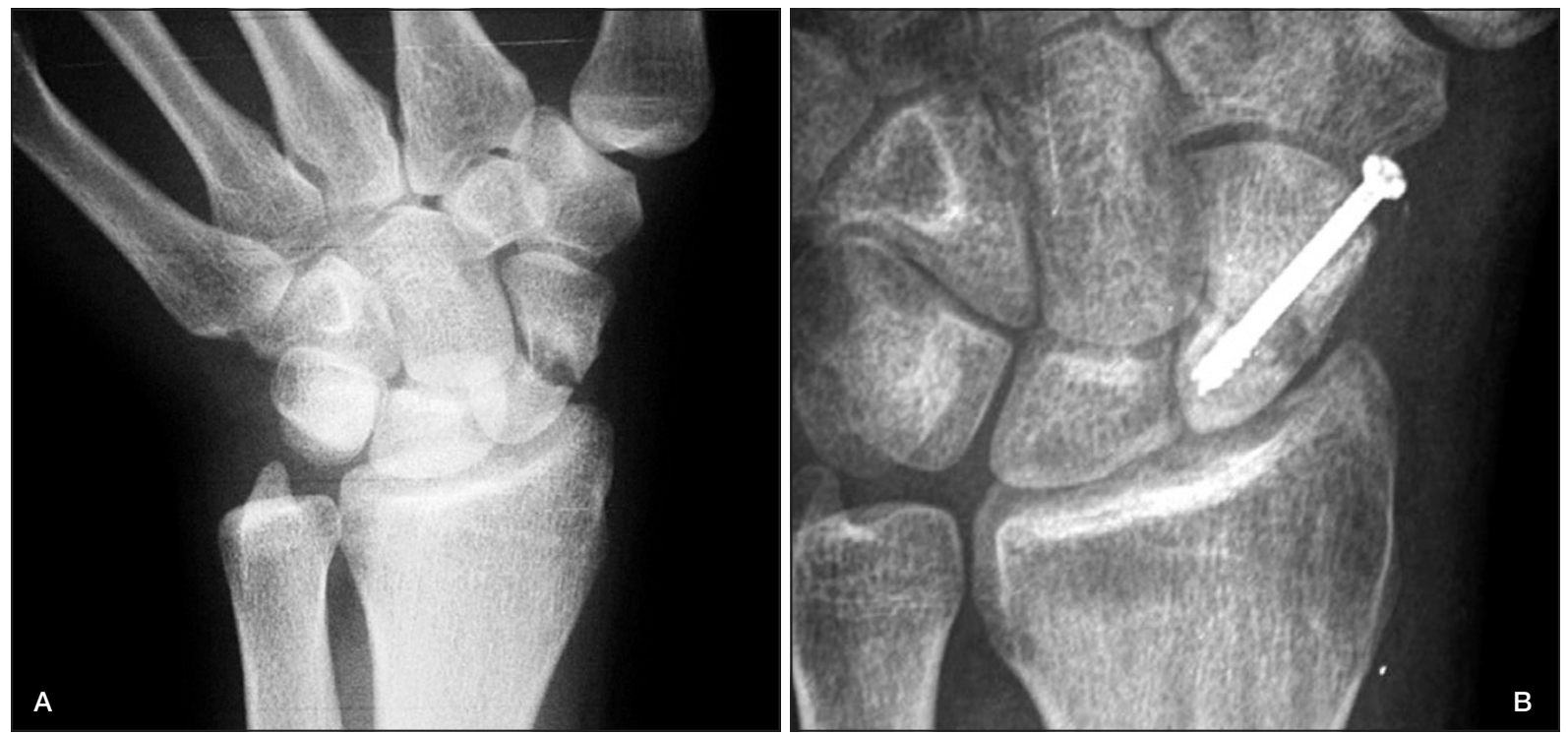

Figura 1. A: Seudoartrosis de tercio medio IIA de Alnot ${ }^{8}$, imagen preoperatoria. B: Resultado a las 12 semanas. Nótese que el tornillo no está perfectamente centrado en el polo proximal y es largo, lo que impide una compresión correcta. Retraso de consolidación en paciente asintomático.

cambios adaptativos, con un mayor tiempo para conseguir la consolidación y con una menor recuperación del balance articular ${ }^{1,6,13-16}$. Otro factor que influye en la rapidez de consolidación está en relación con la correcta colocación de la cabeza del tornillo en el fragmento proximal. En concreto, aquellos que quedan centrados y siguen el eje longitudinal del escafoides consiguen antes el objetivo gracias a una osteosíntesis más estable ${ }^{1,13}$ (Figuras 1 y 2). Nagle ${ }^{17}$ prefiere el uso de múltiples aK para evitar la dificultad técnica que supone una correcta colocación del tornillo y lograr una estabilidad parecida, pero recomienda inmovilizar tres meses. Kawamura y Chung ${ }^{14}$ y Merrell et al. ${ }^{18}$, en sendas publicaciones, concluyen que los tornillos son mejores que las aK. Munk y Larssen ${ }^{19}$, en una revisión bibliográfica desde 1928 a 2003 constatan que los implantes no mejoran sustancialmente el porcentaje de éxitos, pero sí ayudan a minorar el tiempo de inmovilización al conseguir una importante estabilidad y permitir al hueso solicitaciones mecánicas prematuras que no serían posibles sin el implante.

En los trabajos revisados no queda claro el concepto de consolidación; ¿se considera éxito aquellos casos asintomáticos tras la cirugía pero con radiología en la que no hay certeza de consolidación?, ¿cómo confirmar con total certeza que la seudoartrosis ha consolidado? Todos estos problemas diagnósticos ocurren por ser el escafoides un hueso sin periostio, que precisa de múltiples proyecciones y el material de osteosíntesis supone un porcentaje importante de la sección del hueso. Además, hemos de considerar la interferencia en la señal por culpa del material de osteosíntesis, lo que añade mayor dificultad de interpretación en pruebas de imagen como la TAC. Días ${ }^{20}$ define consolidación cuando podemos comprobar en proyecciones (lo más idénticas posible) sucesivas que no hay signos radiológicos de aflojamiento del implante, desplazamiento de las agujas/injerto intercalado o reabsorción del injerto. Si todos los datos radiológicos antes mencionados están presentes a los seis meses, el autor considera que hay consolidación. En ocasiones estos datos radiológicos se dilatan en el tiempo. Para Días, si no hay clínica ni impotencia funcional y no aparecen datos radiológicos de mal pronóstico se puede hablar también de consolidación en fechas próximas, y podemos incluir al paciente en nuestra casuística de éxitos aunque tardemos un año en ver una radiología satisfactoria. Autores como Barton ${ }^{21}$ refieren que la aparición de puentes óseos en el lado cubital/ dorsal en las Rx se puede considerar una señal de 

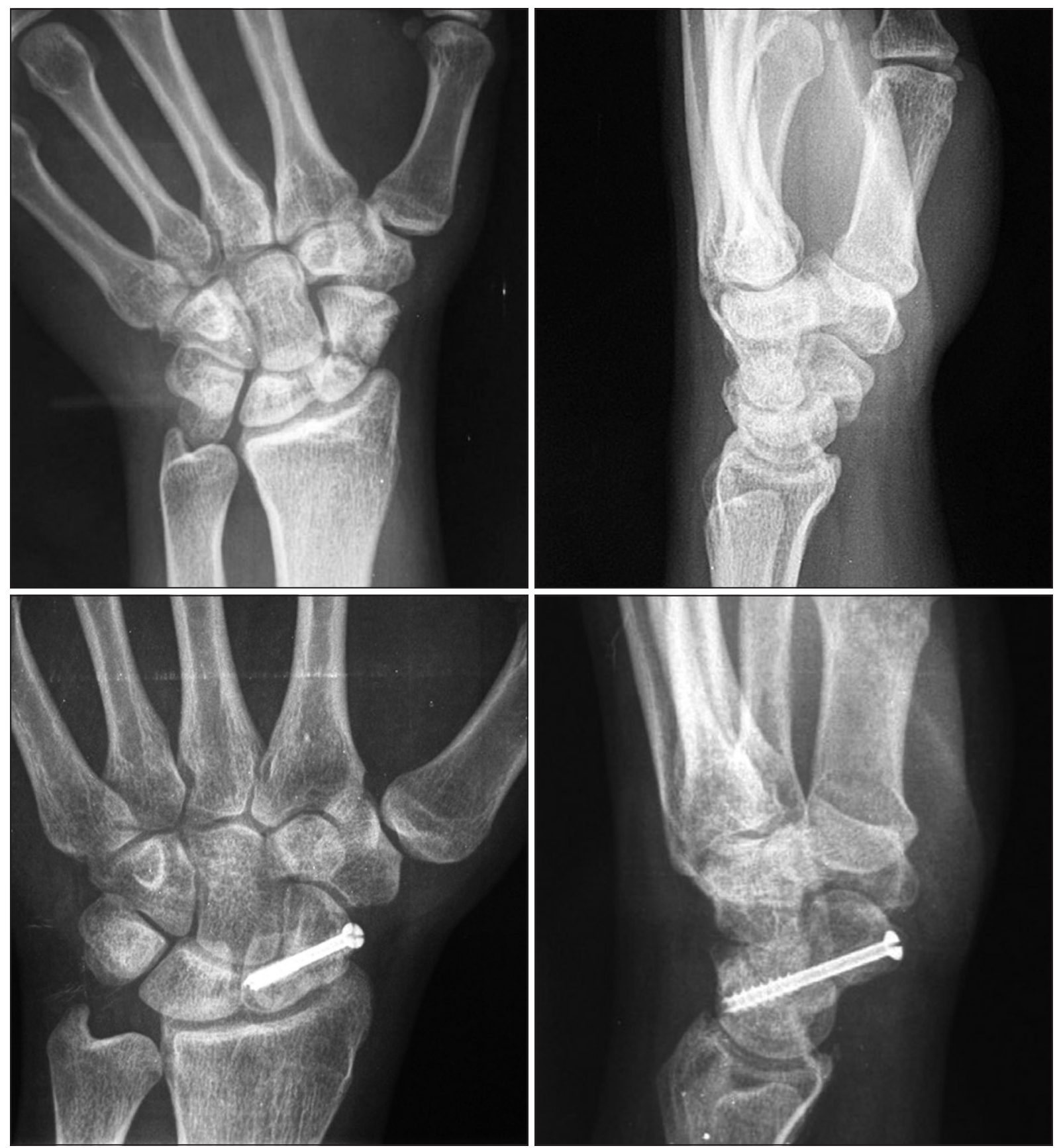

Figura 2. A y B: Seudoartrosis de tercio medio IIA de Alnot ${ }^{8}$, imagen preoperatoria. C y D: Resultado al año.

consolidación en próximas radiografías. Frente a esta corriente, el grupo europeo multicéntrico de Schuind, Haentjens, García-Elías et al. ${ }^{15}$ considera estos mismos casos como fracaso. Todos los autores antes mencionados proponen la realización de una TAC como «ayuda diagnóstica» en casos de duda. Nuevamente, la suma de radiología y exploración física-semiológica nos orientará en la decisión de si existe unión del foco de seudoartrosis.

Tsuyuguchi et al. ${ }^{21}$ encontraron una asociación significativa entre los mejores resultados y una corrección de $10^{\circ}$ o más en el ángulo escafolunar. Trumble et al. ${ }^{13}$ también consiguen un $90 \%$ de éxitos en aquellas SE con menos de 5 años de evolución. Igualmente, Merrell et al. ${ }^{18}$ 
refieren descensos del porcentaje de éxitos si la cirugía se dilata más de 12 meses y Schuind et al. ${ }^{15}$ en su estudio multicéntrico de 138 casos refieren, tras análisis multivariante, que el tiempo de evolución es determinante en el número de éxitos.

\section{CONCLUSIONES}

El número de casos que nuestra serie incorpora no permite realizar grandes estudios estadísticos, pero es muy similar a otros trabajos, el tipo de población estudiada es también muy similar y nos permite extraer varias conclusiones:

Se ha obtenido un porcentaje alto de consolidaciones, en consonancia con otras series similares. Este alto porcentaje de consolidaciones, acompañado de la reincorporación al trabajo habitual y ausencia de progresión de cambios radiológicos justifican el tratamiento quirúrgico. Además, con la técnica de F-F se consigue una mejoría estadísticamente significativa en el ángulo escafolunar; un seguro de no hallar artrosis intracarpiana en un futuro.

En la serie, sólo dos pacientes exceden los cinco años entre el traumatismo y tratamiento quirúrgico. Podemos concluir que el tratamiento quirúrgico precoz minora los cambios adaptativos-artrósicos del carpo y permite mejor correc- ción de éstos si se han iniciado, aumentando el porcentaje de éxitos.

El material de osteosíntesis no tiene relevancia en el resultado final, habida cuenta que en la serie existe un $36 \%$ de pacientes en los que se han empleado aK y en el $64 \%$ restante tornillos canulados (tampoco existe diferencia entre los dos modelos utilizados). Sin embargo, tiene especial relevancia una correcta colocación del material de osteosíntesis en el fragmento proximal. Así, en el $87 \%$ de los casos de fracaso o retraso en la consolidación podemos ver un defecto de técnica, con incorrecta colocación del material de osteosíntesis en el fragmento proximal o distracción-insuficiente compresión del fragmento proximal.

El tiempo medio de consolidación fue menor para la técnica de F-F. Creemos que el motivo del mayor éxito de la técnica de F-F estriba en la estabilidad intrínseca del injerto trapezoidal encastrado a presión ${ }^{23}$, capaz de mejorar el ángulo escafolunar; el único marcador de la gravedad de la SE que en la serie es estadísticamente significativo. Otro factor a favor de los mejores resultados de la técnica de F-F residen en un mayor poder osteoformador-osteoinductor del injerto córticosponjoso, además de un soporte que permite mejor compresión entre los fragmentos al aplicar el material de osteosíntesis ${ }^{23}$.

\section{BIBLIOGRAFÍA}

1. Jupiter JB, Shin AY, Trumble $\mathrm{TE}$, et al. Traumatic and reconstructive problems of the scaphoid. AAOS Inst Course Lect, 2001; 50: 105-22.

2 Linström G, Nyström A. Natural history of scaphoid nonunion, with special reference to «asymptomatic» cases. J Hand Surg Br, 1992; 17: 697-700.

3 Mack GR, Bosse MJ, Gelberman $\mathrm{RH}$. The natural history of scaphoid non-union. J Bone Joint Surg Am, 1984; 66: 50409.

4 Ruby LK, Stinson J, Belsy MR. The natural history of scaphoid non-union. J Bone Joint Surg Am, 1985; 67: 428-32.
5 Kerluke L, McCabe SJ. Nonunion of the scaphoid: A critica analysis of recent natural history studies. J Hand Surg Am, 1993; 18: 1-3.

6 Smith BS, Cooney WP. Revision of failed bone grafting for nonunion of the scaphoid. Treatment, options and results. Clin Orthop, 1996; 327: 98-109.

7 Cooney WP, Linscheid RL, Dobyns $\mathrm{JH}$, et al. Scaphoid nonunion: Role of anterior interpositional bone graft. J Hand Surg Am, 1988; 13: 635-50.

8 Alnot JY. Fractures et pseudoarthroses du scaphoïde carpien. Rev Chir Orthop, 1988; 74: 683-752.
9 Watson K, Ballet FL. The SLAC wrist. Scapholunate advanced collapse pattern of degenerative arthritis. J Hand Surg Am, 1984; 9: 358-65.

10 Krakauer JB, Bishop AT, Cooney WP. Surgical treatment of scapholunate advanced collapse. J Hand Surg Am, 1994; 19: 751-9.

11 Morimoto H, Tada K, Yoshida T, et al. The relationship between the site of nonunion of the scaphoid and scaphiod nonunion advanced collapse (SNAC). J Bone Joint Surg Br, 1999; 81: 871-76.

12 Herbert, TJ. Natural history of scaphoid non-union: A critical 
analysis. Letters to editor. J Hand Surg Am, 1994; 19: 155.

13 Trumble TE, Salas P, Barthel T, et al. Mangement of scaphoid nonunions.. J Am Acad Orthop Surg, 2003; 11: 380-91.

14 Kawamura K, Chung KC. Treatment of scaphoid fractures and nonunions. J Hand Surg Am, 2008; 33: 988-97.

15 Schuind F, Haentjens P, Van Innis $F$, et al. Prognostic factors in the treatment of carpal scaphoid nonunions. J Hand Surg Am, 1999; 24: 761-76.

16 Ramamurthy C, Cutler L, Nuttall $D$, et al. The factors affecting non-vascular bone grafting and internal fixation for nonunion of the scaphoid. J Bone Joint Surg Br, 2007; 89: 627-32.

17 Nagle DJ. Scaphoid nonunion. Treatment with cancellous bone graft and kirschner-wire fixation. Hand Clin, 2001; 17: 625-29.

18 Merrell GA, Wolfe S W, Slade JF III. Treatment of scaphoid nonunions: quantitative metaanalysis of the literature. J Hand Surg Am, 2002; 27: 685-91.

19 Munk B, Larsen C F. Bone grafting the scaphoid nonunion. Acta Orthop Scand, 2004; 75: 618-29.

20 Dias JJ. Definition of union after acute fracture and surgery for fracture nonunion of the scaphoid. J Hand Surg Br, 2001; 26: 321-25.

21 Barton, NJ. Apparent and partial nonunion of the scaphoid. J Hand Surg Br, 1996; 21: 496500.

22 Tsuyuguchi Y, Murase T, Hidaka $\mathrm{N}$, et al. Anterior wedge-shaped graft for old sacphoid fractures or non-unions. J Hand Surg Br, 1995; 20: 194-200.

23 Fernández DL. A technique for anterior wedge-shaped grafts for scaphoid nonunions with carpal instability. J Hand Surg Am, 1984; 9: 733-37. 